\title{
A Spectroscopic Monte-Carlo Model to Simulate the Response of Pixelated CdTe based Detectors
}

\author{
KAL Koch-Mehrin ${ }^{\mathrm{a}, *}$, JE Lees ${ }^{\mathrm{a}}$, SL Bugby ${ }^{\mathrm{a}, \mathrm{b}}$ \\ ${ }^{a}$ Space Research Centre, Department of Physics $\&$ Astronomy, University of Leicester, LE1 7RH, UK \\ ${ }^{b}$ Centre for Sensing and Imaging Science, Department of Physics, Loughborough University, LE11 \\ 3TU, UK
}

\begin{abstract}
A fully spectroscopic Monte Carlo model has been developed to predict the spectroscopic performance of pixelated CdTe based detectors. The model incorporates photon attenuation by the photoelectric effect, Compton scattering and Rayleigh scattering. Charge transport equations are used to simulate the size of the electron cloud, approximated by a symmetrical two-dimensional Gaussian distribution, as it drifts to be read out at the detector anode. Direct comparisons are made between simulated data and an experimentally acquired spectra from a $1 \mathrm{~mm}$ thick CdTe sensor coupled to the HEXITEC detector ASIC. The probability of an absorbed photon leading to charge sharing across pixels as a function of incoming photon energy is investigated. The charge cloud size was found to be dominated by cloud growth during drift for photon energies $<100 \mathrm{keV}$. Furthermore, the portion of charge sharing events due to fluorescence from within the CdTe sensor is calculated - these events are distinguished from regular charge sharing events since their energy response differs. The model described is shown to give a good estimate of the total probability of charge sharing for energies up to $140 \mathrm{keV}$. CdTe sensor thickness, bias voltage, pixel size and electronic noise threshold can be adjusted to model a range of detector architectures.

Keywords: Monte Carlo detector model, CdTe, HEXITEC, Charge Sharing, Pixelated compound semiconductor detectors
\end{abstract}

\footnotetext{
* Corresponding author

Email address: kalkm1@le.ac.uk (KAL Koch-Mehrin)
} 
2

\section{Introduction}

Semiconductor radiation detectors based on compounds such as CdTe offer direct Xray detection due to a high effective atomic number $(\mathrm{Z}=50)$ and density $\left(\sim 5.85 \mathrm{~g} / \mathrm{cm}^{3}\right)$. Furthermore, CdTe's large bandgap results in low leakage current and noise [1], which in turn allows operation at room temperature with good spectral performance [2] without the need for cooling devices. For these reasons, CdTe sensor detector systems can be designed to achieve relatively conservative volume and power consumption, making them attractive candidates for hard X-ray and soft gamma-ray imaging and spectroscopy in nuclear medicine [3] and astronomy [4] applications.

Pixelating CdTe detectors has the obvious advantage of allowing imaging and, if the pixel pitch is small compared to the sensor thickness, mitigating the effects of poor hole mobility with the small pixel effect [5]. Material impurities and defects limit the charge carrier mobilities in these materials, particularly for holes, resulting in incomplete charge collection [1] and polarisation effects [6], thereby ultimately a reduction in spectroscopic performance. The small pixel geometry ensures that a significant charge is only induced by charge carriers drifting close to the anode pixel, described by the Schockley-Ramo theorem [7]. If the detector is back-illuminated such that the majority of interactions occur far from the anode, the detector will effectively become single carrier sensing in favour of the electrons by minimising the induced signal from the easily trapped holes, leading to better spectroscopic performance.

However, the downside to small pixel array detectors is that their performance suffers due to increased amounts of charge sharing [8]. Charge sharing occurs when the electron charge cloud from a single absorbed incident photon induces a charge across multiple pixels. The extent to which charge sharing occurs for these events, which will be referred to as shared events, depends on the initial charge cloud size, charge diffusion, Coulomb repulsion, X-ray fluorescence and Compton scattering. The process behind the formation of shared events is therefore quite complex, depending both on electron transport and secondary photon effects. Analytical models based on electron transport equations, which consider the geometrical and physical parameters of the detector and pixel array, have been used to estimate the amount of charge sharing across neighbouring pixels 8, 9, 10, 11. In some cases these work well, but they do not factor in the influence of 
the noise threshold or fluorescence photons on charge sharing, which have been shown to be significant [12]. In order to model stochastic effects such as X-ray fluorescence from the sensor material, Compton recoil electrons, photon pile-up and the distribution of electrons in a charge cloud, Monte-Carlo methods are needed. This has motivated the development of an end-to-end fully spectroscopic Monte-Carlo model, which can simulate the response of a pixelated CdTe detector for single pixel events and multipixel shared events.

In this work, the Monte Carlo model is described in detail and tested against experimental data obtained from observations using a $1 \mathrm{~mm}$ thick CdTe detector coupled to the HEXITEC ASIC [13]. The probability of an absorbed photon resulting in charge sharing is investigated as a function of the incoming photon's energy. Factors which influence the probability of charge sharing in pixelated CdTe detectors are discussed. Furthermore, charge sharing events that are due to photon fluorescence are distinguished from regular charge sharing events, and the relative incidence of each compared to experimental values.

\section{Model}

The Monte-Carlo model has been developed in the Python programming language 14] and can simulate the response of a pixelated CdTe detector for incoming photon energies ranging from 1 to $1000 \mathrm{keV}$. When the model is run, the interaction position of the incoming photon is determined by considering the photoelectric effect, Compton scattering and Rayleigh scattering. Secondary emissions such as X-ray fluorescence and Compton electrons are also considered. Once the interaction position is known, the electron transport of the charge cloud (approximated to be a two-dimensional Gaussian) as it drifts from its interaction position to be read-out at the anode is computed. The number of pixels the charge cloud spreads across, and how the charges are distributed amongst those pixels, is determined, as well as any charge loss due to charge trapping. The information containing the amount of charge collected in each pixel is stored in an event list, which is compiled from every photon (and its secondary emissions) introduced into the simulation. The event list can then be processed in the same manner as experimental data in order to reconstruct a spectrum for each event-type. 


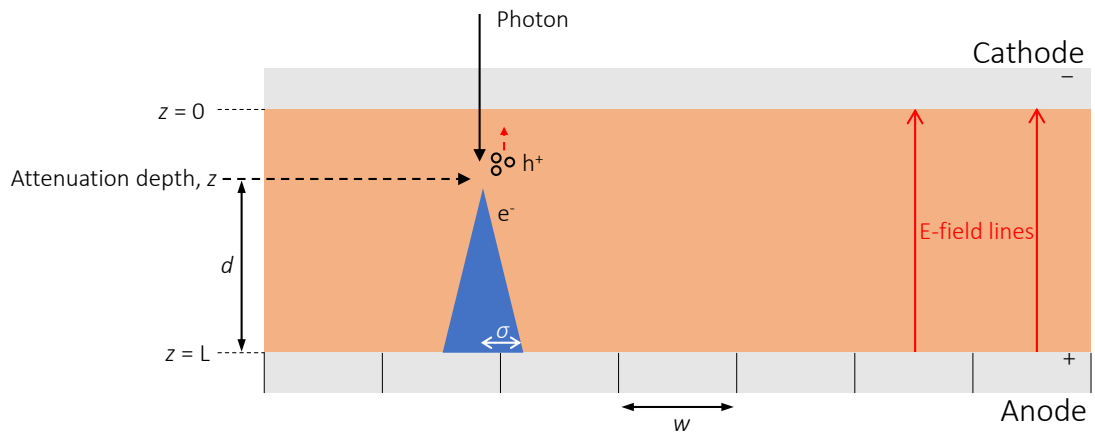
variables used in the schematic are referred to throughout this section.

Figure 1: Schematic of the CdTe detector as simulated by the model. The electrodes are positioned above and below the CdTe sensor in the middle. The entire CdTe sensor is assumed to be depleted by the electric field. The cathode is planar whereas the anode is pixelated. Although the cathode is drawn here, its attenuation is assumed negligible by the model. The thickness of the sensor is given by $L$ and $d$ is the drift length of the electrons to the anode. The electron charge cloud, as it is the one that is computed, is represented by a cone which increases in diameter as it moves towards the anode and spreads its charges over more than one pixel. The width of the Gaussian charge cloud (i.e. standard deviation) is given by $\sigma$.

Figure 1 shows a schematic of how the detector is simulated within the model. The

\section{deviation) is given by}

\subsection{Photon attenuation}

The number of primary photons and their energy, $E$, is specified by the user. The photons are modelled as uniformly illuminating across the entire collecting area through the cathode and are assumed to be travelling perpendicular to the detector surface. Any attenuation by the cathode is taken to be negligible. The z-axis is defined to be from the cathode to the anode, measured from the surface of the sensor on the cathode side.

The attenuation depth, $z$, into the sensor from the cathode side for each photon is computed from the probability distribution obtained from the Beer-Lambert law 15

$$
P(z)=e^{-\mu(E) z}
$$

where $\mu$ is the total linear attenuation coefficient of CdTe and depends on the energy $E$ of the photon. The total linear attenuation coefficient is given by summing the sepa- 

98 is 18

rate attenuation coefficients of the photoelectric effect, Compton scattering and Rayleigh scattering. Values for $\mu$ are taken from the National Institute of Standards and Technology (NIST) XCOM database [16], interpolated on a log-log scale. Photons for which $z$ is greater than the thickness of the sensor pass through the sensor unattenuated and are consequently removed from the simulation.

\subsubsection{Scattering}

The relative attenuation cross-sections [16] at the photon energy $E$ are used to determine the type of attenuation at position $z$. For the Photoelectric effect for example, the relative attenuation $P_{\mu_{p e}}$ is

$$
P_{\mu_{p e}}=\frac{\mu_{p e}(E)}{\mu(E)}
$$

where $\mu_{p e}$ is the attenuation coefficient for the photoelectric effect only. If attenuation occurs via the photoelectric effect, the primary photon is assumed to be completely absorbed at depth $z$. Otherwise, the photon is scattered by either Rayleigh or Compton scattering (determined by the relative attenuations). The scattered photons either interact at a new position, or escape the sensor and are removed from the simulation. This step is repeated until all scattered photons have either been absorbed in the sensor or escaped. The distance the scattered photons travel before interacting again is calculated using equation 1 .

In the case of Rayleigh scattering, since it is a form of coherent scattering, the scattered photon does not transfer any of its energy to an electron. Instead, only the direction of travel of the photon changes. The possible scattering angles are defined by a probability distribution that depends on the energy $E$ of the photon being scattered and the $Z$ number of the atom involved in the interaction [17]. Since $Z$ will depend on whether the photon interacts with a $\mathrm{Cd}$ or Te atom, the atom the photon interacts with is first determined. The probability, $P$, of the photon interacting with a Cd atom, for example,

$$
P=W_{C d} \frac{\left(\frac{\mu}{\rho}\right)_{C d}}{\left(\frac{\mu}{\rho}\right)_{C d T e}}
$$


122 jump factor, $J$, 18 16.

\subsection{Absorption}

where $W_{C d}$ is the weight fraction of $\mathrm{Cd}$ in $\operatorname{CdTe}(0.468)$ and $\rho$ the density of the material

When Compton scattering occurs, the primary photon imparts some of its energy to the electron it interacts with and is scattered in a new direction. The energy of the scattered photon, $E^{\prime}$, is defined by 19

$$
E^{\prime}=\frac{E}{1+\frac{E}{m_{e} c^{2}}(1-\cos \theta)}
$$

where $\theta$ is the scattering angle, $m_{e}$ the electron mass and $c$ the speed of light. Compton scattered photons are assumed only to interact with free electrons. The Klein-Nishina formula [20] is therefore used to determine $\theta$ from the probability distribution function of all possible scattering angles for a photon with energy $E$. The energy absorbed by the Compton scattered electron at the interaction position is then

$$
E_{e}=E-E^{\prime}
$$

At this stage, all primary and scattered photons have either been absorbed via the photoelectric effect or escaped the sensor. The energy a primary or scattered photon deposits at the interaction position is dependent on the atom and shell it is absorbed by, and any resulting fluorescence photons.

The atom the photon interacts with is determined using equation 3 . The shell the photon interacts with will first depend on the binding energy of the shells relative to the energy of the photon. If the energy of the photon does not exceed the binding energy $E_{b}$ of a shell, absorption by that shell is not possible. The binding energy of the shells are taken from 21. Where $E>E_{b}$, the shell the photon is absorbed by is determined by comparison of the mass attenuation coefficients of the individual shells. Electrons bound to the $\mathrm{K}, \mathrm{L}_{1}, \mathrm{~L}_{2}$ and $\mathrm{L}_{3}$ shells are considered in the model. The mass attenuation for an individual shell can be calculated from the total mass attenuation of the atom and its

$$
\left(\frac{\mu}{\rho}\right)_{\text {shell }}=\frac{J-1}{J}\left(\frac{\mu}{\rho}\right)_{t o t}
$$


The jump factor is the fraction of the total photoelectric absorption coefficient due to absorption by electrons of the respective shell.

\subsubsection{Fluorescence}

A vacancy is created in the shell that the photon interacts with. The fluorescence yield for the respective shell is used to determine if the filling of the vacancy by a cascading electron results in a fluorescence photon. If fluorescence does not occur, the complete energy of the primary photon is assumed to be absorbed by the photoelectron such that $E_{e}=E$. If a fluorescence photon is emitted from the atom, the relative radiative rates are used to determine which transition has occurred. Possible transitions, their line energies and their relative rates for both $\mathrm{Cd}$ and Te are shown in table 1 21. A vacancy that forms in an L-shell of the same atom due to a $K \alpha$ transition, may also fluoresce and that fluorescence photon may then escape the atom too. The energy of the photoelectron $E_{e}$, where fluorescence does occur, is therefore

$$
E_{e}=E-\sum_{i=1}^{n} E_{f l_{i}}
$$

where $E_{f l_{i}}$ is the energy of the fluorescence photon from the $i$ th shell. Vacancies that arise in an $\mathrm{M}$ or $\mathrm{N}$ shell due to the cascading of electrons or otherwise, are ignored. Given that the binding energy for these shells is very small (e.g. $0.618 \mathrm{keV}$ for $\mathrm{M}_{3}$ shell in $\mathrm{Cd}$ 21]), and that any resulting fluorescence will consequently be of very low energy (0.603 $\mathrm{keV}$ for $M_{\gamma}$ line [22]) and is therefore most likely to be reabsorbed close to the original vacancy (mean path length of $1 \mathrm{keV}$ in CdTe is $0.2 \mu \mathrm{m}$ ), the energy from these shells is assumed not to escape the pixel and is instead included in the primary photoelectron energy.

The direction of the fluorescence photons is uniformly distributed over all solid angles. The steps described so far for the primary photons are repeated for the secondary fluorescence photons and any further fluorescence photons that result from those. When absorption by $\mathrm{K}$ and $\mathrm{L}$ shells can no longer occur (i.e. when the energy of all remaining photons is less than the binding energy of the $\mathrm{Cd} \mathrm{L}_{3}$ shell, $3.537 \mathrm{keV}$ [21]), the remaining photons are assumed to be absorbed in either an $\mathrm{M}$ or $\mathrm{N}$ shell (if they have not escaped the sensor). 


\begin{tabular}{ccccc}
\hline \multirow{2}{*}{ Transition } & \multicolumn{3}{c}{$\mathrm{Te}$} \\
\cline { 2 - 5 } & Line energy $(\mathrm{keV})$ & Rate & Line energy $(\mathrm{keV})$ & Rate \\
\hline$K \alpha_{1}\left(\mathrm{KL}_{3}\right)$ & 23.174 & 0.55 & 27.473 & 0.53 \\
$K \alpha_{2}\left(\mathrm{KL}_{2}\right)$ & 22.984 & 0.29 & 27.202 & 0.29 \\
$K \beta_{1}\left(\mathrm{KM}_{3}\right)$ & 26.095 & 0.09 & 30.996 & 0.10 \\
$K \beta_{2}\left(\mathrm{KM}_{2}\right)$ & 26.654 & 0.02 & 31.700 & 0.03 \\
$K \beta_{3}\left(\mathrm{KM}_{2}\right)$ & 26.061 & 0.05 & 30.945 & 0.05 \\
\hline$L \alpha_{1}\left(\mathrm{~L}_{3} \mathrm{M}_{5}\right)$ & 3.134 & 0.77 & 3.769 & 0.75 \\
$L \alpha_{2}\left(\mathrm{~L}_{3} \mathrm{M}_{4}\right)$ & 3.127 & 0.08 & 3.758 & 0.08 \\
$L \beta_{2}\left(\mathrm{~L}_{3} \mathrm{~N}_{4}\right)$ & 3.528 & 0.12 & 4.302 & 0.14 \\
$L l\left(\mathrm{~L}_{3} \mathrm{M}_{1}\right)$ & 2.767 & 0.03 & 3.336 & 0.03 \\
\hline$L \beta_{1}\left(\mathrm{~L}_{2} \mathrm{M}_{4}\right)$ & 3.317 & 0.91 & 4.030 & 0.88 \\
$L \gamma_{1}\left(\mathrm{~L}_{2} \mathrm{~N}_{4}\right)$ & 3.717 & 0.09 & 4.571 & 0.12 \\
\hline$L \beta_{3}\left(\mathrm{~L}_{1} \mathrm{M}_{3}\right)$ & 3.401 & 1.00 & 4.120 & 1.00 \\
\hline
\end{tabular}

Table 1: Possible transitions for $\mathrm{Cd}$ and Te atoms used in the model, along with line energies and relative radiative rates for each transition 21 rounded to three and two significant figures.

\subsection{Charge transport}

At this stage, the initial position of each photoelectron from absorbed primary and secondary photons is known. The photoelectron will lose energy by ionisation, creating electron-hole pairs along its path until it is stopped. The number of electron-hole pairs, $N$, is given by

$$
N=\frac{E_{e}}{\omega}
$$

where $\omega$ is the average pair creation energy, equal to $4.3 \mathrm{eV}$ in $\mathrm{CdTe}$ [1]. The variance on $N$ is described by the Fano-noise and is assumed to follow Fano adjusted Poisson statistics $\left(\sigma_{N}^{2}=F N\right)$. The Fano factor, $F$, is taken to be 0.1 for CdTe [23]. 


\subsubsection{Charge cloud size}

The charge cloud is modelled as a symmetrical two dimensional Gaussian distribution 24, 25] perpendicular to the direction of the electric field lines (i.e. the z-axis). The final size of the cloud will depend on the initial size, $\sigma_{i}$, from the excitation of electronhole pairs by the photoelectron and the growth of the cloud, $\sigma_{d}$, due to diffusion and electrostatic repulsion of the charges as the cloud drifts to the anode. The size of the charge cloud $\sigma$ at the anode is described by adding the two components in quadrature within the assumption that their magnitudes are independent of one another

$$
\sigma=\sqrt{\sigma_{d}^{2}+\sigma_{i}^{2}}
$$

The cloud growth due to diffusion, $\sigma_{d}$, can be calculated using the diffusion equation derived from Fick's second law [26]

$$
\frac{\partial \sigma_{d}(t)^{2}}{\partial t}=2 D
$$

where $D$ is the diffusion coefficient and $\sigma_{d}(t)^{2}$ is a function of time. The diffusion coefficient is given by the Einstein relation [27.

$$
D=\mu_{e} \frac{k_{b} T}{q}
$$

where $\mu_{e}$ is the electron mobility, $k_{b}$ Boltzmann's constant, $q$ the elementary charge and $T$ the temperature of the sensor. The total drift time $t_{d}$ for the electron cloud to reach the anode is equal to

$$
t_{d}=d \frac{L}{\mu_{e} V}
$$

where $L$ is the thickness of the CdTe sensor, $d$ the drift length of the electron cloud to the anode (given by $L-z$ ) and $V$ the applied bias voltage. The electrostatic repulsion during drift is included by using an effective diffusion constant $D^{\prime}[24$ in place of $D$ in equation 10

$$
D^{\prime}=D+\frac{\mu_{e} N q}{24 \pi^{3 / 2} \epsilon} \frac{1}{\sigma(t)}
$$


where $\epsilon$ is the permittivity in CdTe and $\sigma(t)$ is the size of the electron cloud after some arbitrary amount of time $t$. By combining equations 10,11 and 13 , the differential equation which gives the size of the electron cloud due to diffusion and repulsion is

$$
\frac{\partial \sigma_{d}(t)^{2}}{\partial t}=\frac{2 \mu_{e} k_{b} T}{q}+\frac{\mu_{e} N q}{12 \pi^{3 / 2} \epsilon} \frac{1}{\sigma(t)}
$$

A numerical solution is found for $\sigma_{d}$ by using the 4th order Runge-Kutta method over $10^{3}$ time steps between $t=0$ and $t=t_{d}$. At $t=0$, the size of the electron cloud $\sigma(t)$ is assumed to be equal to $\sigma_{i}$. Once equation 14 has been solved, equation 9 is used to compute the final size of the electron cloud.

GEANT simulations by Blevis and Levinson [28] give estimates for the initial charge cloud size generated by a photoelectron at different energies, which have previously been used in Monte-Carlo simulations to model X-ray spectra from a CdTe detector [25. The values used for $\sigma_{i}$ in this work are $0 \mu \mathrm{m}, 1 \mu \mathrm{m}, 4 \mu \mathrm{m}$ at electron energies of $0 \mathrm{keV}, 35$ $\mathrm{keV}$ and $100 \mathrm{keV}$. A second order polynomial fit is used to extract $\sigma_{i}$ for each absorbed photoelectron energy $E_{e}$ in the simulation.

\subsubsection{Charge collection efficiency}

As charges drift to an electrode, they can become trapped in defects and impurities in the crystal resulting in charge loss and therefore incomplete charge collection. To account for this effect, the Hecht relation [29], which calculates the charge collection efficiency $(C C E)$, is used

$$
C C E=\frac{\lambda_{h}}{L}\left(1-e^{-\frac{\psi(z)}{\lambda_{h}}}\right)+\frac{\lambda_{e}}{L}\left(1-e^{-\frac{\psi(L-z)}{\lambda_{e}}}\right)
$$

where $\lambda_{h}=\mu_{h} \tau_{h} E_{f}, \lambda_{e}=\mu_{e} \tau_{e} E_{f}$ are the mean drift lengths of holes and electrons respectively, and are a product of the carrier lifetime $\tau$, the carrier mobility $\mu$ and the electric field strength $E_{f}$. The electric field is assumed to be uniform across the sensor (i.e. an effective electric field strength is used to account for a non-uniform field, see section 4.1). The weighting potential, $\psi$, takes into consideration the geometry of the sensor and correctly describes the relative current that the respective charge carrier can induce on the anode. Figure 2 shows the weighting potential for the HEXITEC CdTe detector for which $w / L=0.25$, where $w$ is the size of the pixel pitch 30]. The curve for 

small pixel geometry.

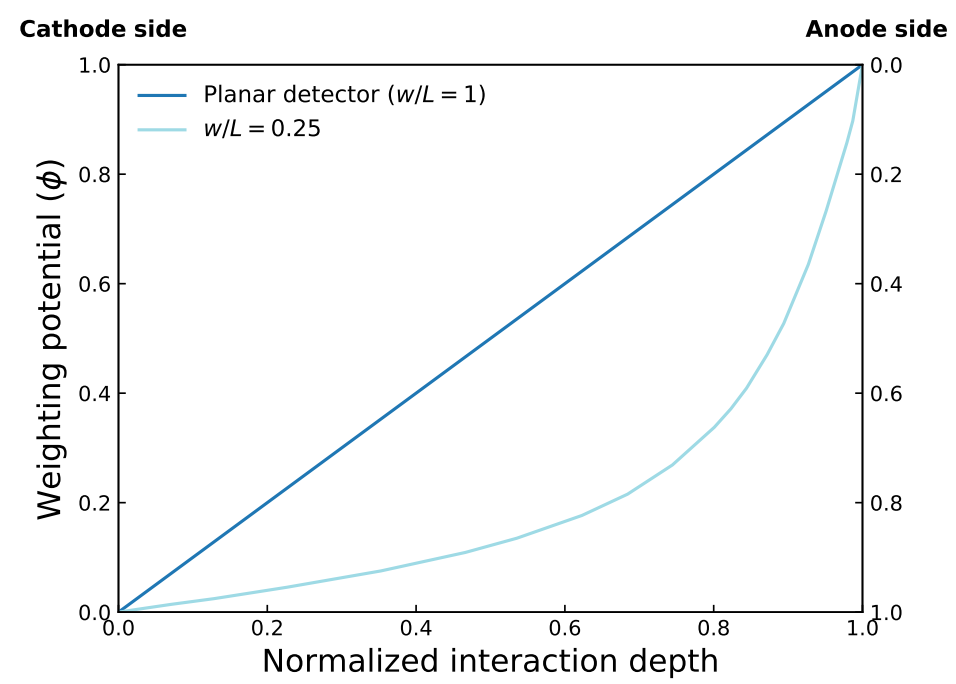

Figure 2: Weighting potential for a planar detector $(w / L \geq 1)$ and for the geometry of the HEXITEC CdTe sensor. The left-hand y-axis is the weighting for the holes and therefore the cathode side. The right-hand $\mathrm{y}$-axis is the weighting for the electrons and therefore the anode side.

a planar detector where the pixel size is equal to or greater than the sensor thickness is shown for comparison. The weighting potential therefore describes the effect of the

The $C C E$ is calculated for each electron charge cloud by first sampling the weighting potential at the interaction depth $z$ and then using equation 15 The effective number of charges that is equivalent to the current that is actually induced on the anode, $N^{\prime}$, is then

$$
N^{\prime}=C C E \times N
$$

\subsubsection{Pixel readout}

For each charge cloud, a two-dimensional Gaussian distribution with standard deviation $\sigma$, containing $N^{\prime}$ charges, is evaluated against the pixelated array, centred on the absorption position. The charges in each pixel are summed to obtain the energy deposited in that pixel. The model does not simulate charge loss across the inter-pixel region. 
Electronic noise is added to each pixel that contains an energy. The electronic noise is assumed to follow a Gaussian distribution with variance $\sigma^{2}=A$ where $\mathrm{A}$ is equal to the equivalent noise charge (ENC). The ENC is a free parameter in the model that can be adjusted to match experimental data.

An eventlist containing the charge collected in each pixel for every frame in the simulation is saved. Secondary emissions are assumed to happen instantaneously and are therefore recorded in the same frame as the primary photon which triggered them.

\subsection{Spectral response reconstruction}

The structure of the eventlist generated by the model is identical to the format in which the experimental data from the HEXITEC ASIC is recorded. Simulated event lists are therefore processed using the analysis algorithm for experimental data described by Bugby et al 12 .

The algorithm classifies the type of each event. Pixels that are adjacent to one another are said to be part of the same event. The event type then depends on the number of pixels the event contains - the considered event types are shown in figure 3 . The energy of an event is the sum of the energy in all pixels of that event. A separate spectrum is reconstructed for each event type, where only events with a shape as it is shown in figure 3 are included, such that linear tripixels and quadpixels for example (which were more likely to exhibit photon pileup) are not added to their event type spectrum. Different orientations for identically shaped events showed no difference in their energy response and are therefore considered as the same event type. Diagonally adjoined events are counted as two separate events [12].

It is common practise to apply a noise threshold to the energy in each pixel of an observation in order to remove counts that are due to thermal charge leakage from the crystal and detector electronics, instead of an absorbed photon. The noise threshold is applied by the algorithm - removing all pixels in an event that have an energy lower than the threshold.

\section{Detector description}

The detector system used in this work is the STFC High Energy X-ray Imaging Technology (HEXITEC) [13, developed to detect hard X-rays and gamma rays. The 


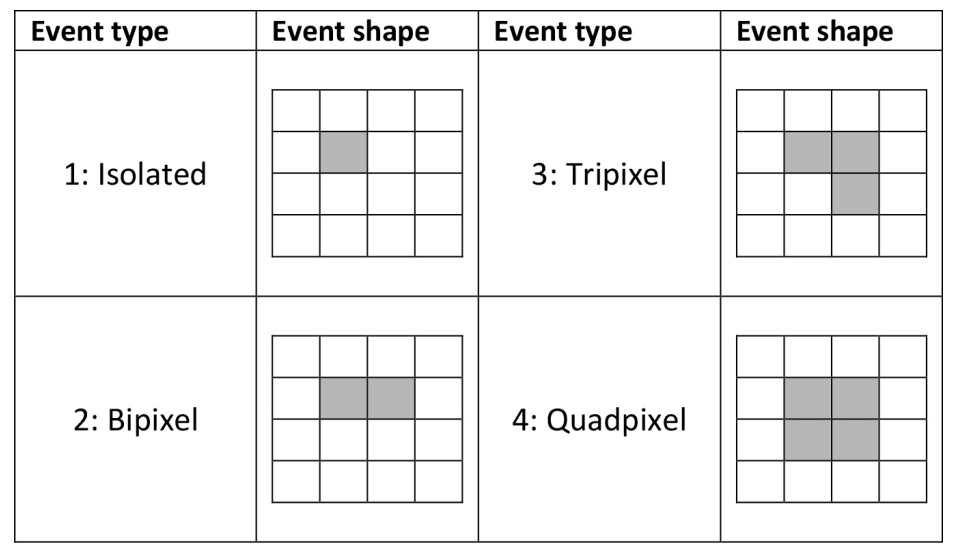

Figure 3: Events shapes for isolated, bi-, tri- and quad- pixel events. The greyed shaded squares correspond to pixels which have recorded an energy greater than the noise threshold.

HEXITEC consists of a detector that is coupled to an ASIC to perform spectroscopic observations. The ASIC is pixelated by an $80 \times 80$ array with a pitch of $250 \mu \mathrm{m}$ manufactured on a standard $0.35 \mu \mathrm{m}$ CMOS (complementary metal-oxide semiconductor) process, such that each ASIC pixel contains a charge amplifier, shaping amplifier and a peak track-and-hold circuit. Therefore, induced charge is converted to a voltage at the pixel level, allowing each pixel to provide a full energy spectrum. The design of the detector consists of a platinum planar cathode and pixelated aluminium anode on opposite sides of a CdTe semiconductor material. The CdTe sensor has a thickness of 1 $\mathrm{mm}$ and a $20 \times 20 \mathrm{~mm}$ collecting area. The anode is pixelated by an $80 \times 80$ array with a pixel electrode size of $200 \mu \mathrm{m}$ and an inter-pixel spacing of $50 \mu \mathrm{m}$. The ASIC and anode are attached by flip-chip assembly to gold studs placed on each of the ASIC bond pads.

The HEXITEC system has shown good spectral result room temperature with a CdTe sensor [31, achieving a mean FWHM energy resolution for all pixels of $0.75 \mathrm{keV}$ for incoming photons of energy $59.5 \mathrm{keV}$.

\section{Data collection}

\subsection{Simulated data}

For each simulation, the model was run with $10^{7}$ primary photons and with a sensor temperature of $28^{\circ} \mathrm{C}$. 
Cola et al. show that when applying a bias voltage of $-500 \mathrm{~V}$, the effective voltage is in fact $-425 \mathrm{~V}$ because of a nonuniform electric field across the sensor thickness which decreases with distance from the anode 32 . Test simulations were performed using the nonuniform electric field profile shown in 32 when applying a $-500 \mathrm{~V}$ bias. However, comparative simulations using an equivalent effective bias voltage and a uniform field produced the same results. Therefore, in the interest of reducing computation time, the effective value is used for the bias voltage in the simulations.

Since the model does not distinguish between the inter-pixel gap and the pixel electrode, the pixel size used in the simulations is the electrode size of $200 \mu \mathrm{m}$ on a $80 \times 80$ pixel array. Charge induction maps show that electric field lines at the anode still detect electrons from the inter-pixel region, with the field lines from the gap's neighbouring pixels sharing the inter-pixel region equally 33 .

The ENC was set to 60 electrons rms in order to match the FWHM of the simulated photopeaks to that of the experimental photopeaks for isolated events. An energy bin size of $0.3 \mathrm{keV}$ and a noise threshold of $3 \mathrm{keV}$ were used.

\subsection{Experimental data}

The energy response in each pixel of the detector was calibrated using the methods previously reported in [12. Table 2 lists the sources and line energies used for calibration.

As K-shell X-ray fluorescence is known to have an effect on charge sharing in CdTe [8], peaks closer to the absorption edges of CdTe $(\sim 26-31 \mathrm{keV})$ were desirable. An X-ray tube (Amptek Mini-X [35]), was used to obtain additional photopeaks could be obtained at $32.5 \mathrm{keV}, 38.0 \mathrm{keV}$ and $45.8 \mathrm{keV}$. The tip of the Amptek X-ray tube was placed $3 \mathrm{~cm}$ from the surface of the detector and equipped with a $2 \mathrm{~mm}$ collimator. A silver (Ag) transmission target was used to obtain the three photopeaks, with electrons accelerated at the target across a tube voltage of $35 \mathrm{kV}, 40 \mathrm{kV}$ and $50 \mathrm{kV}$. Since only photons above the CdTe absorption edges were of interest, Niobium (Nb), Gold ( $\mathrm{Au}$ ) and Chromium (Cr) filters were used to remove lower energy photons. Therefore, peaks were effectively created from the bremsstrahlung continuum emitted from the X-ray tube. Table 3 shows the additional peaks acquired from the X-ray tube measurements and the filter(s) used, and table 4 gives details on the filters. 


\begin{tabular}{ccccc}
\hline Source & $\begin{array}{c}\text { Source activity } \\
(\mathrm{MBq})\end{array}$ & $\begin{array}{c}\text { Photopeak energies } \\
(\mathrm{keV})\end{array}$ & $\begin{array}{c}\text { Acquisition time } \\
(\mathrm{s})\end{array}$ & $\begin{array}{c}\text { Frame occupancy } \\
(\%)\end{array}$ \\
\hline${ }^{109} \mathrm{Cd}$ & 84.9 & $24.90 \pm 0.01$ & 1000 & 0.4 \\
& & $88.00 \pm 0.01$ & 0.5 \\
\hline${ }^{241} \mathrm{Am}$ & 351.7 & $59.54 \pm 0.01$ & 1000 & 0.2 \\
\hline${ }^{57} \mathrm{Co}$ & 51.7 & $14.40 \pm 0.10$ & & 0.2 \\
\hline${ }^{55} \mathrm{Fe}$ & 414 & $122.10 \pm 0.05$ & 7000 & 0.4 \\
\hline${ }^{99 \mathrm{~m}} \mathrm{Tc}$ & 188.3 & $136.50 \pm 0.10$ & & \\
\hline
\end{tabular}

Table 2: Photopeak energies obtained from experimental flood images taken by 12 using different radioisotope sources. Multiple peaks (i.e. $\mathrm{K}_{\alpha 1}$ and $\mathrm{K}_{\alpha 2}$ ) have been combined into a single peak energy taking into account their relative intensity where these cannot be resolved. The range of energies included in each photopeak is shown as a standard error, this is set to a nominal value of $10 \mathrm{eV}$ when individual peaks have not been combined. The frame occupancy is the mean percentage of pixels in each frame which are above the noise threshold [34].

Figure 4 shows the event type spectra for the $40 \mathrm{kV}$ and $50 \mathrm{kV}$ tube voltage acquisitions. It can be seen that the bremsstrahlung peaks for all event types are well separated from the lower energy peaks coming from the Ag X-ray tube target and Nb filter K-shell fluorescence in the case of the $50 \mathrm{kV}$ observation. This meant that charge sharing at the energy of the main photopeak could be analysed without any interference, identically to the radioisotope source peaks. However, since the X-ray tube acquired photopeaks are not from a discrete energy as for the radioisotope peaks, and have a FWHM of $\sim 7 \mathrm{keV}$, the uncertainty on the energy for the peaks acquired from these observations is \pm 3.5 $\mathrm{keV}$.

For all acquisitions, the detector was run with a bias voltage of $-500 \mathrm{~V}$ and the detector temperature was observed to remain at $28 \pm 1^{\circ} \mathrm{C}$. The bias voltage was periodically refreshed by switching the bias to $0 \mathrm{~V}$ for 2 seconds every 60 seconds. This was done 


\begin{tabular}{ccccccccc}
\hline \multirow{2}{*}{$\begin{array}{c}\text { Tube voltage } \\
(\mathrm{kV})\end{array}$} & $\begin{array}{c}\text { Tube current } \\
(\mu \mathrm{A})\end{array}$ & $\begin{array}{c}\text { Photopeak energy } \\
(\mathrm{keV})\end{array}$ & $\begin{array}{c}\text { Acquisition time } \\
(\mathrm{s})\end{array}$ & $\begin{array}{c}\text { Frame occupancy } \\
(\%)\end{array}$ & \multicolumn{2}{c}{ Filter(s) used } \\
\cline { 6 - 9 } & & & & & $\mathrm{Au}$ & $\mathrm{Cr}$ & $\mathrm{Nb}$ \\
\hline 35 & 110 & $32.5 \pm 3.5$ & 1800 & 0.2 & $\mathrm{X}$ & & \\
40 & 80 & $38.0 \pm 3.5$ & 1800 & 0.2 & $\mathrm{X}$ & $\mathrm{X}$ & \\
50 & 5 & $45.8 \pm 3.5$ & 1200 & 0.2 & & & $\mathrm{X}$ \\
\hline
\end{tabular}

Table 3: Photopeak energies obtained from X-ray tube measurements by accelerating the electrons with different electric field strengths. The uncertainty of the photopeak energies is determined from the peak FWHM. The applied filter(s) is marked by an X.

\begin{tabular}{ccccc}
\hline \multirow{2}{*}{ Filter } & \multirow{2}{*}{$\begin{array}{c}\text { Density } \\
\left(\mathrm{g} / \mathrm{cm}^{3}\right)\end{array}$} & $\begin{array}{c}\text { thickness } \\
(\mathrm{mm})\end{array}$ & \multicolumn{2}{c}{ Fluorescence X-ray emission $(\mathrm{keV})$} \\
\cline { 4 - 5 } & & $\mathrm{K}_{\alpha}$ & $\mathrm{K}_{\beta}$ \\
\hline $\mathrm{Au}$ & 19.32 & 0.25 & $68.12 \pm 0.10$ & $77.98 \pm 0.01$ \\
$\mathrm{Cr}$ & 7.19 & 1.00 & $5.41 \pm 0.05$ & $5.95 \pm 0.01$ \\
$\mathrm{Nb}$ & 8.57 & 1.00 & $16.50 \pm 0.10$ & $18.62 \pm 0.01$ \\
\hline
\end{tabular}

Table 4: The different filters used for the X-ray tube measurements shown in table 3 The K-shell fluorescence photon emission energies from the filters is also shown. The $K_{\alpha 1}$ and $K_{\alpha 2}$ peaks were combined and errors were determined in the same way as for the photopeaks in table 2

to prevent known time-dependent polarisation effects of Schottky contact CdTe [6]. To minimise the likelihood of shared events occurring due to photon pileup, it was ensured that the frame occupancy in each observation did not exceed $1 \%$.

The spectral reconstruction algorithm described in Section 2.4 was used on the raw experimental data files to build the energy spectra. An energy bin size of $0.3 \mathrm{keV}$ and a noise threshold of $3 \mathrm{keV}$ were used.

\section{Comparison with measurement}

\subsection{Simulated spectral detector response}

The Monte-Carlo model was tested against experimental data by simulating the detectors response to an ${ }^{241} \mathrm{Am}$ source and comparing to the experimental spectrum, shown in figure 5. The model can only accurately simulate the peak width for isolated events 


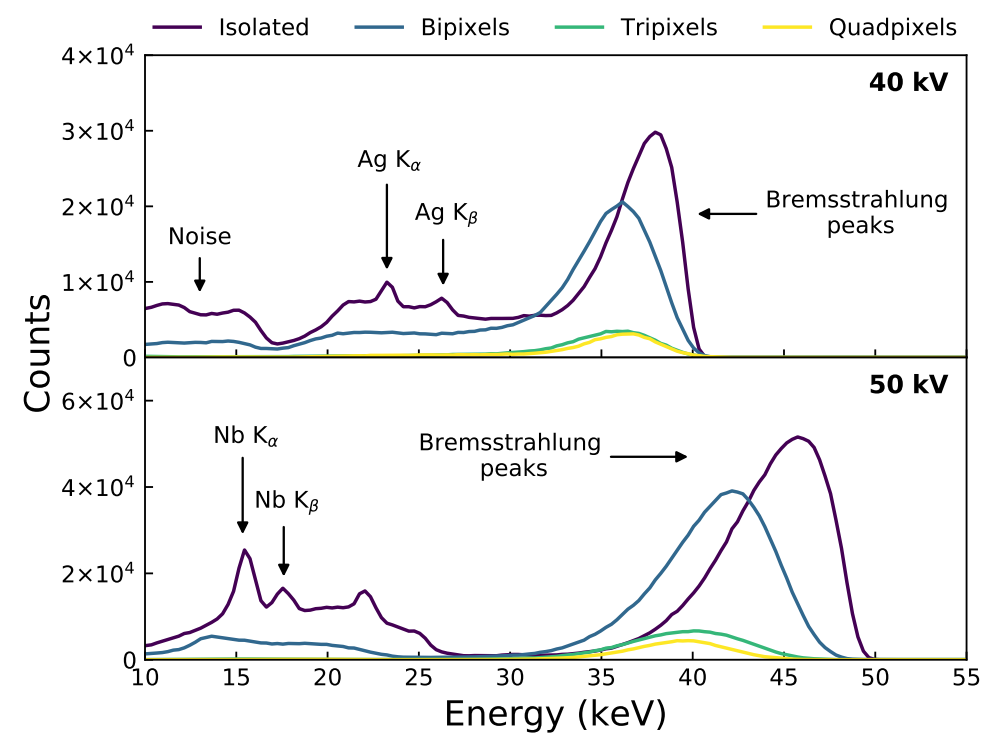

Figure 4: The spectrum for isolated, bipixel, tripixel and quadpixel events detected with the HEXITEC from the $40 \mathrm{kV}$ (Top) electron accelerated X-ray tube acquisition using the Au and Cr filters, and 50 $\mathrm{kV}$ (Bottom) electron accelerated X-ray tube acquisition using the Nb filter. The photopeaks for the multipixel events are positioned at a lower energy due to charge loss to the inter-pixel gap.

as it does not account for the charge loss across the inter pixel gap for shared events. Therefore, only the spectrum from isolated events is compared. The simulated and experimental data are normalised by the maximum number of counts in an energy bin of the primary photopeak.

The detector response to the ${ }^{241} \mathrm{Am}$ source is dominated by the primary gamma emission line at $59.5 \mathrm{keV}$. The lower energy X-ray L-shell lines from the ${ }^{241} \mathrm{Am}$ decay product ${ }^{237} \mathrm{~Np}$ are not observed due to shielding from the $0.5 \mathrm{~mm}$ thick steel source housing. Attenuation by the source housing was also applied to the simulated data. All the peaks seen in figure 5 and their measured positions from experiment and simulation are listed in table 5. The escape peaks are due to fluorescence escaping a pixel which absorbed a $59.5 \mathrm{keV}$ photon, by either leaving the sensor or being reabsorbed beyond the nearest pixel neighbours. The escaped fluorescence photons which are reabsorbed within the sensor lead to the fluorescence peaks at $\sim 23$ and $\sim 26 \mathrm{keV}$. The total number of 59.5 $\mathrm{keV}$ absorbed isolated events is the sum of all counts in the primary gamma photopeak 


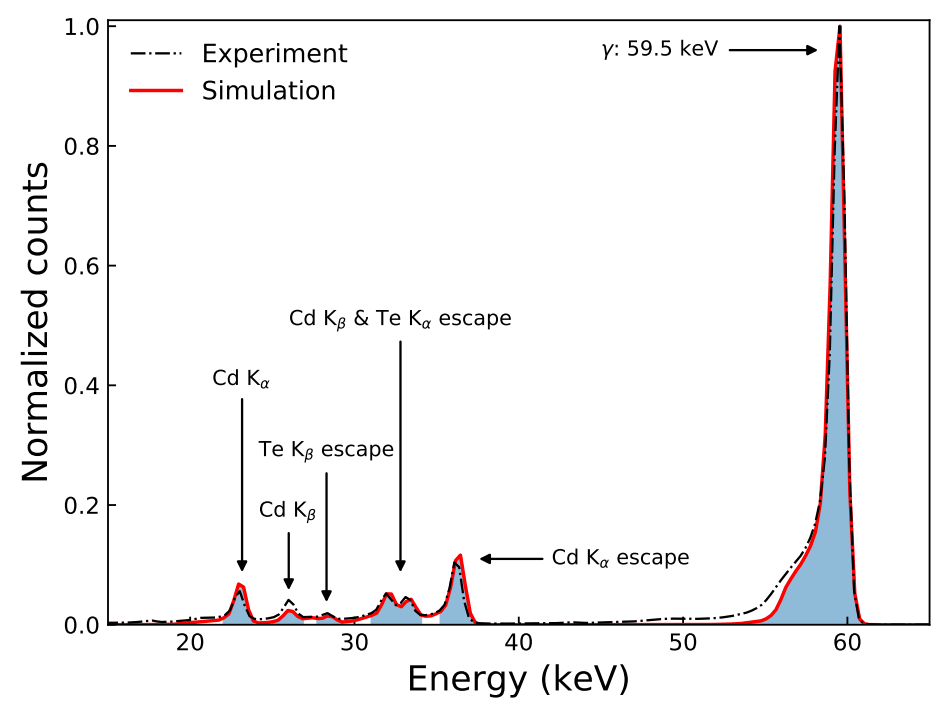

Figure 5: Experimentally observed ${ }^{241} \mathrm{Am}$ spectrum compared with the simulated response to the same source. The spectra are normalised to the maximum counts in an energy bin of the $59.5 \mathrm{keV}$ photopeak and only include isolated events. The shaded regions highlight the energy windows used to calculate the total number of counts belonging to each peak.

$344 \quad 2.3 .2$. 


\begin{tabular}{|c|c|c|c|c|c|}
\hline \multirow{2}{*}{ Peak } & \multicolumn{2}{|c|}{ Peak position (keV) } & \multirow{2}{*}{$\begin{array}{l}\text { Energy Window } \\
\quad(\mathrm{keV})\end{array}$} & \multicolumn{2}{|c|}{ Proportion of counts (\%) } \\
\hline & Experiment & Model & & Experiment & Model \\
\hline$\gamma$ & 59.5 & 59.5 & $50-62$ & $85.0 \pm 1.0$ & $84.0 \pm 0.8$ \\
\hline $\mathrm{Cd} \mathrm{K}_{\alpha}$ escape & 36.2 & 36.4 & $35-38$ & $6.6 \pm 0.3$ & $7.9 \pm 0.2$ \\
\hline $\mathrm{Cd} \mathrm{K}_{\beta}$ escape & 33.1 & 33.4 & $31-34.5$ & $6.7 \pm 0.3$ & $6.7 \pm 0.3$ \\
\hline Te $\mathrm{K}_{\alpha}$ escape & 31.9 & 32.1 & & & \\
\hline $\mathrm{Te} \mathrm{K}_{\beta}$ escape & 28.4 & 28.5 & $27.5-29.5$ & $1.8 \pm 0.4$ & $1.4 \pm 0.3$ \\
\hline $\mathrm{Cd} \mathrm{K}_{\beta}$ & 26.0 & 26.0 & $25-27$ & $3.1 \pm 0.4$ & $1.9 \pm 0.2$ \\
\hline $\mathrm{Cd} \mathrm{K}_{\alpha}$ & 23.0 & 23.0 & $22-24$ & $3.6 \pm 0.2$ & $4.2 \pm 0.1$ \\
\hline
\end{tabular}

Table 5: All visible peaks in the ${ }^{241} \mathrm{Am}$ spectrum and their peak energy positions as measured from the experimental and simulated data shown in figure 5 The energy window corresponds to the shaded region underneath the respective peak in figure 5 The percentages are the proportion of counts in the respective peak from the total number of absorbed incident isolated events (i.e. $\gamma$ photopeak and escape peak counts summed). The errors for the proportion of counts were determined by increasing the energy window by $0.5 \mathrm{keV}$ at both the lower and upper bound and calculating the difference.

\subsection{Shared events}

The probability of a shared event occurring due to charge sharing was investigated as a function of energy from the experimentally acquired data and compared with simulations. The detector response was simulated at multiple energies covering the experimental photopeak energy range from $5.95 \mathrm{keV}$ to $140.5 \mathrm{keV}$. For both experiment and simulation, the event list for each observation was analysed using the reconstruction algorithm described in Section 2.4 - producing a spectrum for each event type of interest. An energy noise threshold of $3 \mathrm{keV}$ was applied.

\subsubsection{Energy response of shared events}

Figure 6 shows the different event type spectra for the ${ }^{109} \mathrm{Cd}$ and ${ }^{57} \mathrm{Co}$ experimentally acquired data. Photopeaks made of multi-pixel event types are much broader than the equivalent isolated event type photopeak. This is due to the effect of charge loss, partially caused by charges drifting in the inter-pixel region [33. The reasons behind charge loss 


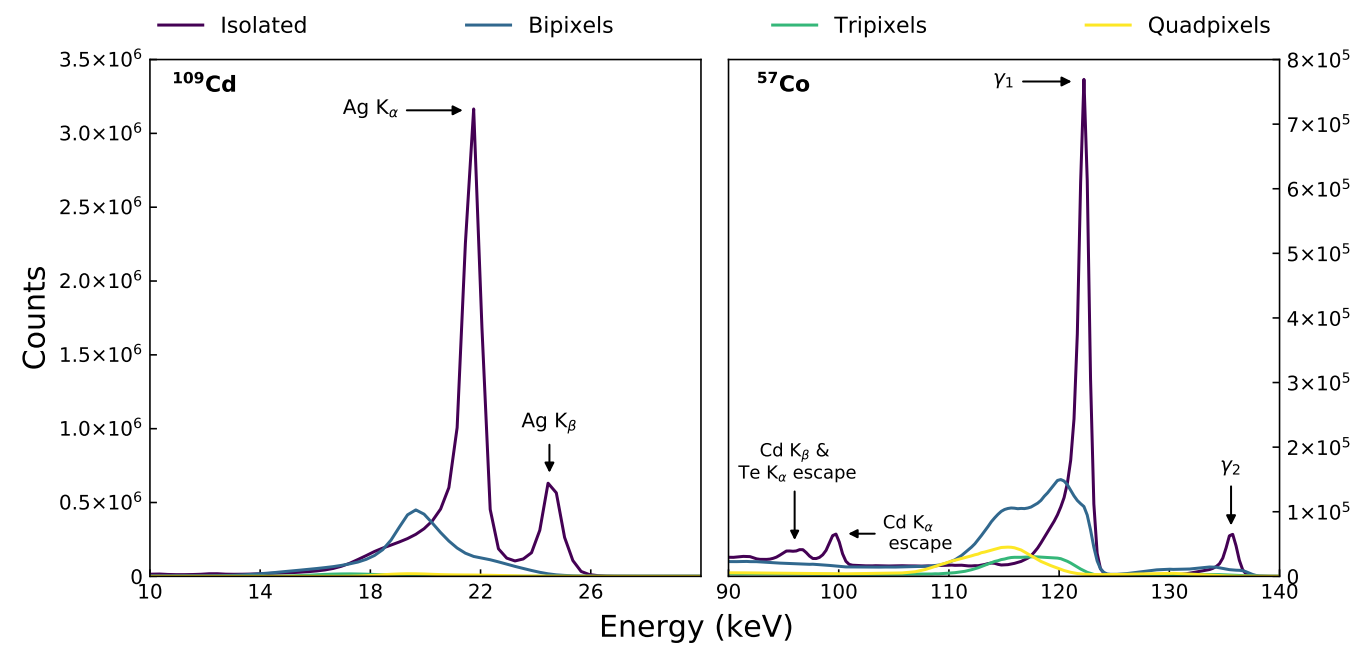

Figure 6: Event type spectra for ${ }^{109} \mathrm{Cd}$ (LHS) and ${ }^{57} \mathrm{Co}$ (RHS) from experimental observations.

across the pixel gaps are described in more detail in [12. However, some charge sharing events do not exhibit as much, if any, charge loss. This can be seen by the $122 \mathrm{keV}$ photopeak $\left({ }^{57} \mathrm{Co}\right.$ source) in the bipixel event spectrum which shows two components - a peak at $121 \mathrm{keV}$ followed by a flat shoulder at lower energies. This is believed to be due to two types of shared events - those created by fluorescence and those solely created by the charges from one cloud centre spreading. In the case of fluorescence shared events, a fluorescence photon escapes into a pixel adjacent to the pixel of the incident photon and deposits its energy there directly. The shared event is not created by charges spreading across the inter-pixel region, as is the case for the other type of shared event, and therefore does not suffer from charge loss. For the $22 \mathrm{keV}$ photons in the ${ }^{109} \mathrm{Cd}$ bipixel spectrum, no two distinct peak components can be seen. Since $\mathrm{K}$-shell fluorescence does not occur at this energy, all of the counts in the ${ }^{109} \mathrm{Cd}$ bipixel photopeak suffer from charge loss, shifting the peak to $19.5 \mathrm{keV}$.

Figure 7 shows the energy split across the two pixels for all $59.5 \mathrm{keV}{ }^{241} \mathrm{Am}$ bipixel events. The straight line indicates the case in which no charge loss occurs - along which summing the energy in the two pixels always equals the known photopeak energy of 59.5 $\mathrm{keV}$. The actual distribution deviates from this line due to the charge loss in the charge spreading shared events. The bright spots in figure 7 correspond to the fluorescence 
shared events. The fluorescence spots are along the straight line, indicating these events do not suffer from charge loss.

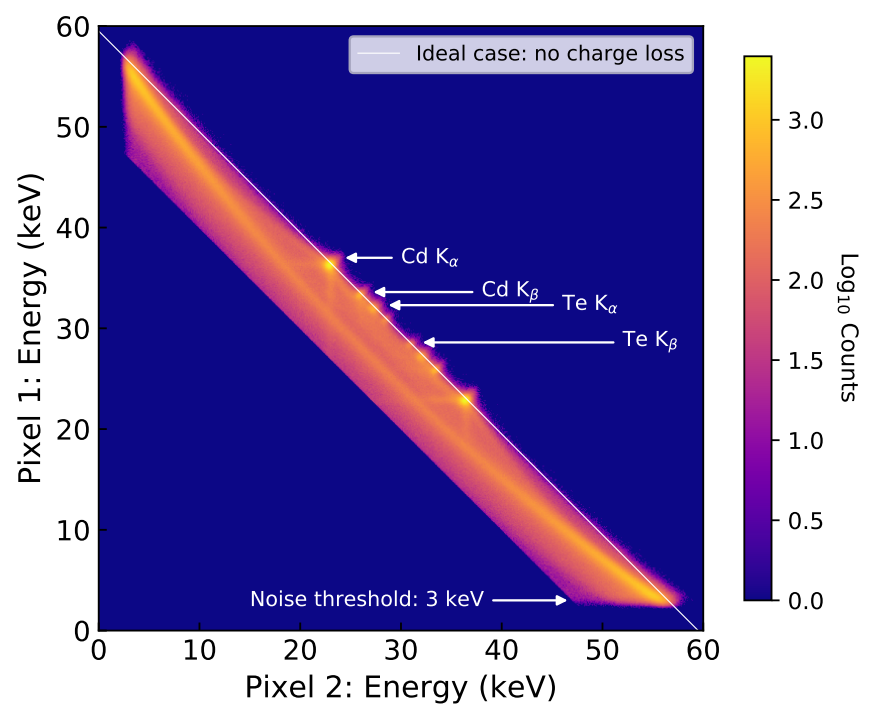

Figure 7: Two-dimensional histogram showing the distribution of split energies for all bipixels in the energy range from $50-62 \mathrm{keV}$ for the ${ }^{241} \mathrm{Am}$ data. The straight yellow line shows the ideal case in which no charge loss occurs. The gaps on either axis from $0-3 \mathrm{keV}$ correspond to the noise threshold. The bright spots are annotated with the fluorescence photon which causes the bipixel event. In total there are eight bright spots - only four are annotated because the remaining four are the same but with the energies in opposite pixels.

\subsubsection{Probability of a shared event}

For both the experimental and simulated data, the likelihood of the event types introduced in figure 3 occurring at a given energy was determined. This was done by using energy windows around the photopeaks of interest and counting the number of events belonging to each specific event type. The number of counts of each event type was normalised by the total number of counts including all event types for the given photopeak.

Figure 8 shows the experimentally calculated probability of any shared event occurring at the incoming photon energies listed in table6, compared with the simulated result at the same energies and some additional energies. The individual probabilities of the 


\begin{tabular}{cccccc}
\hline \multirow{2}{*}{ Source } & Peak energy $(\mathrm{keV})$ & \multicolumn{4}{c}{ Energy window $(\mathrm{keV})$} \\
\cline { 3 - 6 } & & Isolated & Bipixel & Tripixel & Quadpixel \\
\hline${ }^{55} \mathrm{Fe}$ & $5.95 \pm 0.01$ & $4-11$ & $4-11$ & $4-11$ & $4-11$ \\
\hline \multirow{2}{*}{${ }^{109} \mathrm{Cd}$} & $23.10 \pm 1.00$ & $13-28$ & $12-28$ & $13-28$ & $14-28$ \\
& $88.00 \pm 0.01$ & $77-92$ & $75-91$ & $73-91$ & $73-91$ \\
\hline${ }^{241} \mathrm{Am}$ & $59.54 \pm 0.01$ & $39-63$ & $38-63$ & $37-63$ & $37-64$ \\
\hline \multirow{2}{*}{${ }^{57} \mathrm{Co}$} & $14.40 \pm 0.10$ & $9-16$ & $6-16$ & $6-15$ & $11-16$ \\
& $74.00 \pm 1.00$ & $63-77$ & $60-76$ & $59-75$ & $59-73.5$ \\
\hline${ }^{99 m} \mathrm{Tc}$ & $122.10 \pm 0.05$ & $105-126$ & $105-126$ & $105-125$ & $105-124$ \\
\hline X-ray tube $(35 \mathrm{kV})$ & $32.5 \pm 3.5$ & $27-36$ & $25-36$ & $23-36$ & $23-36$ \\
X-ray tube $(40 \mathrm{kV})$ & $38.0 \pm 3.5$ & $28-42$ & $28-42$ & $28-42$ & $28-42$ \\
X-ray tube $(50 \mathrm{kV})$ & $45.8 \pm 3.5$ & $31-51$ & $26-50$ & $24-49$ & $25-48$ \\
\hline
\end{tabular}

Table 6: Photopeak energies at which the probability of charge sharing is calculated. The data and analysis for the peak energies obtained from the radioisotope sources is from 12. The peaks observed from the X-ray tube experiments are from this work. The $74 \mathrm{keV}$ energy analysed from the ${ }^{57} \mathrm{Co}$ acquisition data occurs due to $\mathrm{Pb}$ fluorescence from shielding in the experimental setup. The peak energy uncertainties have been defined in the same manner as in table 2 The ${ }^{109} \mathrm{Cd} 22.00 \mathrm{keV}$ and $24.90 \mathrm{keV}$ peaks have been combined and are treated as a single photopeak with average energy of 23.10 $\mathrm{keV}$ when considering the relative intensities. The energy windows used for each event type for the experimental spectra are also given.

different event types occurring as a function of incoming photon energy, are shown in figure 9 for both the experimentally calculated result and the model prediction. Errors for the event type probabilities are estimated at $\leq 2 \%$ due to Poisson counting statistics and the dependency on the position of the energy window.

Both the experimental result and model prediction shown in figure 8 suggest that the probability of a shared event occurring increases rapidly at lower energies until reaching $50 \%$ at $\sim 50 \mathrm{keV}$. The shared events probability then increases gradually to $62.5 \%$ at 


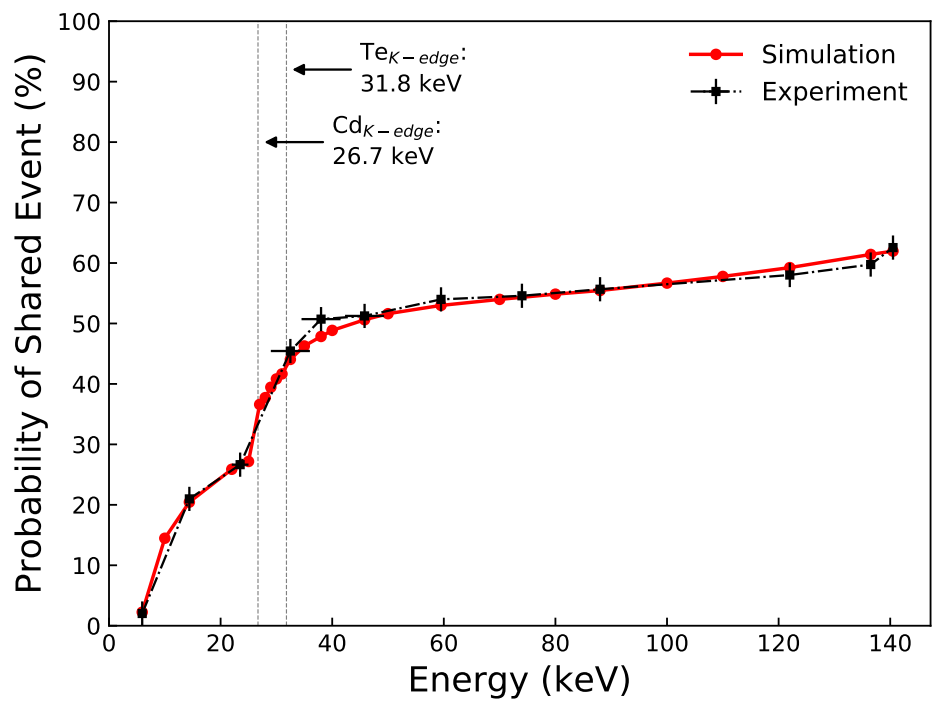

Figure 8: The probability of any type of shared event to occur as a function of incoming photon energy, calculated from experiment and as predicted by the model. The data points mark the energies at which the calculations/simulations were performed. A low noise energy threshold of $3 \mathrm{keV}$ was applied.

$140.5 \mathrm{keV}$.

The trend of the probability of charge sharing with energy is estimated well by the model. The steep increase in shared events at lower energies is due to the noise threshold. At these energies, energy shared with adjacent pixels is more likely to be lower than the threshold, leading to a failure to identify true shared events. The sudden increase at $\sim 26$ $\mathrm{keV}$ (the K-edge of $\mathrm{Cd}$ ) is due to the addition of fluorescence photons. These have mean attenuation ranges of around $100 \mu \mathrm{m}$ and can therefore escape into neighbouring pixels and create shared events. Alternatively, the fluorescence photons may escape beyond the neighbouring pixels, giving rise to the escape peaks. This has the effect of reducing the total number of isolated counts in the main photopeak and therefore also increasing the percentage of shared events at that energy. For the Te K-edge at $\sim 32 \mathrm{keV}$ there is another sudden jump in the shared events probability, although this is much smaller due to the shorter range $(\sim 60 \mu \mathrm{m}$ mean attenuation length) of the Te K-shell fluorescence photons in CdTe. However, the Te K-shell fluorescence photons have energies (27 keV - 


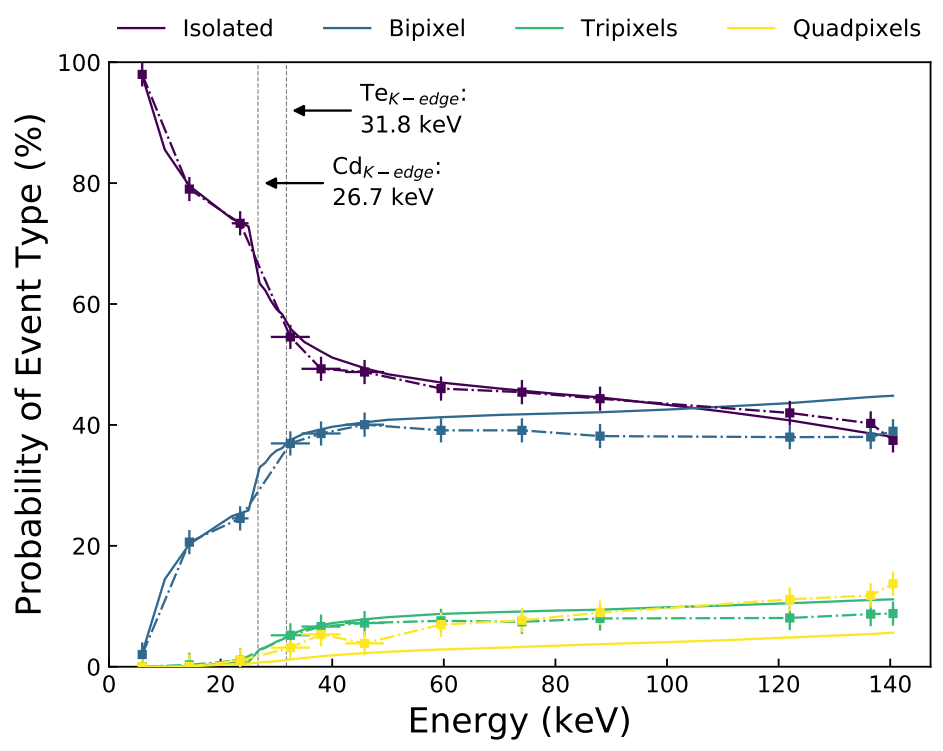

Figure 9: Probability of the individual event type to occur as a function of incoming photon energy. The solid lines are the model prediction, and the dashed lines, with the data points, the experimental result. An low noise energy threshold of $3 \mathrm{keV}$ was applied.

$31 \mathrm{keV}$ ) above the binding energy of the K-shell in Cd. Therefore, if reabsorbed, they can cause additional K-shell fluorescence from the Cd atoms, resulting in pushing the shared events probability up further. Directly after the absorption edges, the shared event probability continues to increase at a relatively steep rate before levelling off. Levelling off is expected to occur at $\sim 35 \mathrm{keV}$, which is where the energy that remains in the pixel after the fluorescence photon escapes, is still above the $3 \mathrm{keV}$ noise threshold. From $\sim 40$ $\mathrm{keV}$ upwards, there is then a gradual increase in the probability of a shared event as the final cloud size becomes increasingly dependent on the width of the initial excitation of charge carries from the photoelectron, rather than on the cloud growth during drift.

figure 9 shows that the majority of the shared events within the investigated energy range are bipixels. Although the total charge sharing probability between the simulated and experimental data agrees very well, the relative contribution of the event types differ at higher energies. The simulated data predicts more bipixels and fewer quadpixels than what is observed experimentally. Furthermore, the simulated results do not show a jump in the probability for quadpixels after the Cd K-edge as suggested by experiment. 


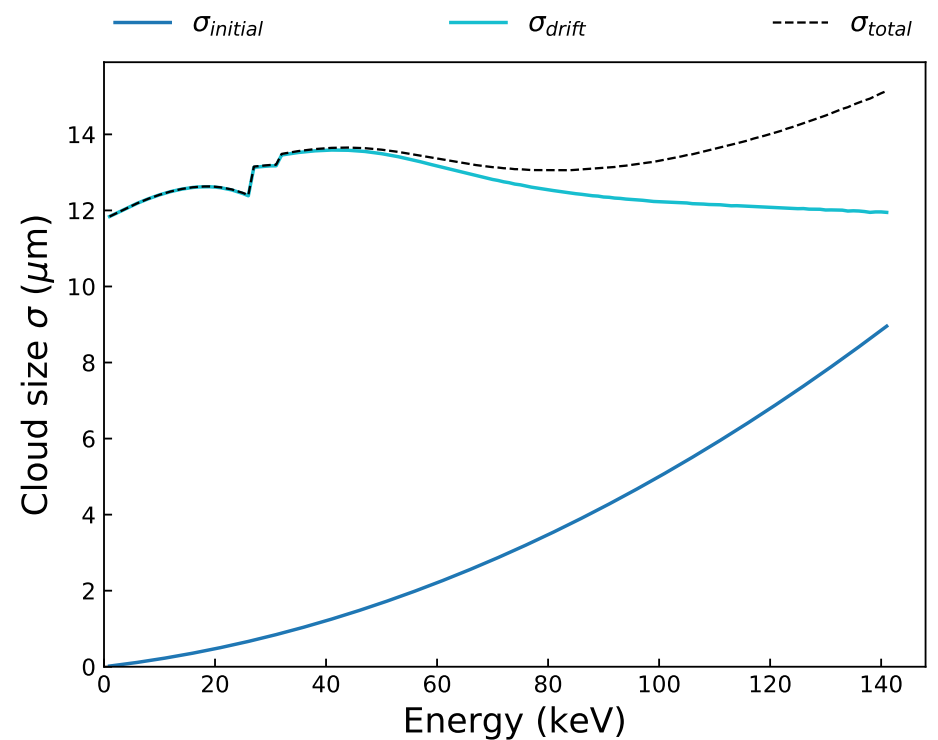

Figure 10: The average charge cloud size at different energies due to the initial width and due to growth during drift to anode as predicted by the model. The combined average total size is also shown. The model was simulated between 1 and $141 \mathrm{keV}$ with $1 \mathrm{keV}$ intervals - only the curve is shown.

Figure 10 shows the average contribution to the final cloud size at the anode in terms of the $\sigma$ of the two distinct components (drift and initial size), as predicted by the model. This suggests that the final cloud size is dominated by the growth due to diffusion and electron repulsion during drift towards the anode up to $\sim 100 \mathrm{keV}$. The average total charge cloud size is found to be relatively constant between 1 and $100 \mathrm{keV}$ at $\sigma \approx 13 \mu \mathrm{m}$. A charge cloud containing $\sim 99.7 \%$ (i.e. $\pm 3 \sigma$ ) of all charges therefore extends $\sim 78 \mu \mathrm{m}$ in diameter. Above the $\mathrm{Cd}$ and $\mathrm{Te} \mathrm{K}$-edges, photons are again absorbed closer to the cathode, increasing the drift time of the excited charges and therewith the cloud size. The relatively constant cloud size supports the argument that at low energies it is the noise threshold that prevents much larger portions of shared events.

\subsubsection{Portion of fluorescence shared events}

In Section 5.2.2 it was shown that fluorescence from the CdTe crystal itself has a considerable impact on the probability of charge sharing. Fluorescence shared events suffer less from charge loss and as a result show better energy resolution and can be 
recombined through simple addition to recover the original photon energy. [12]. It is therefore of interest to quantify the portion of shared events that are due to fluorescence.

Using the data in figure 7 from section 5.2.1, the portion of bipixels at $59.5 \mathrm{keV}$ that are only due to fluorescence were calculated. This was done using two-dimensional energy windows around the bright fluorescence spots in the image, summing the number of counts in those regions and normalising by the total number of bipixels. The same plot and analysis was performed using simulated data at $59.5 \mathrm{keV}$. Furthermore, both experimental and simulated calculations for the portion of fluorescence shared events were repeated for the $122 \mathrm{keV}$ line. Table 7 shows the results from these calculations.

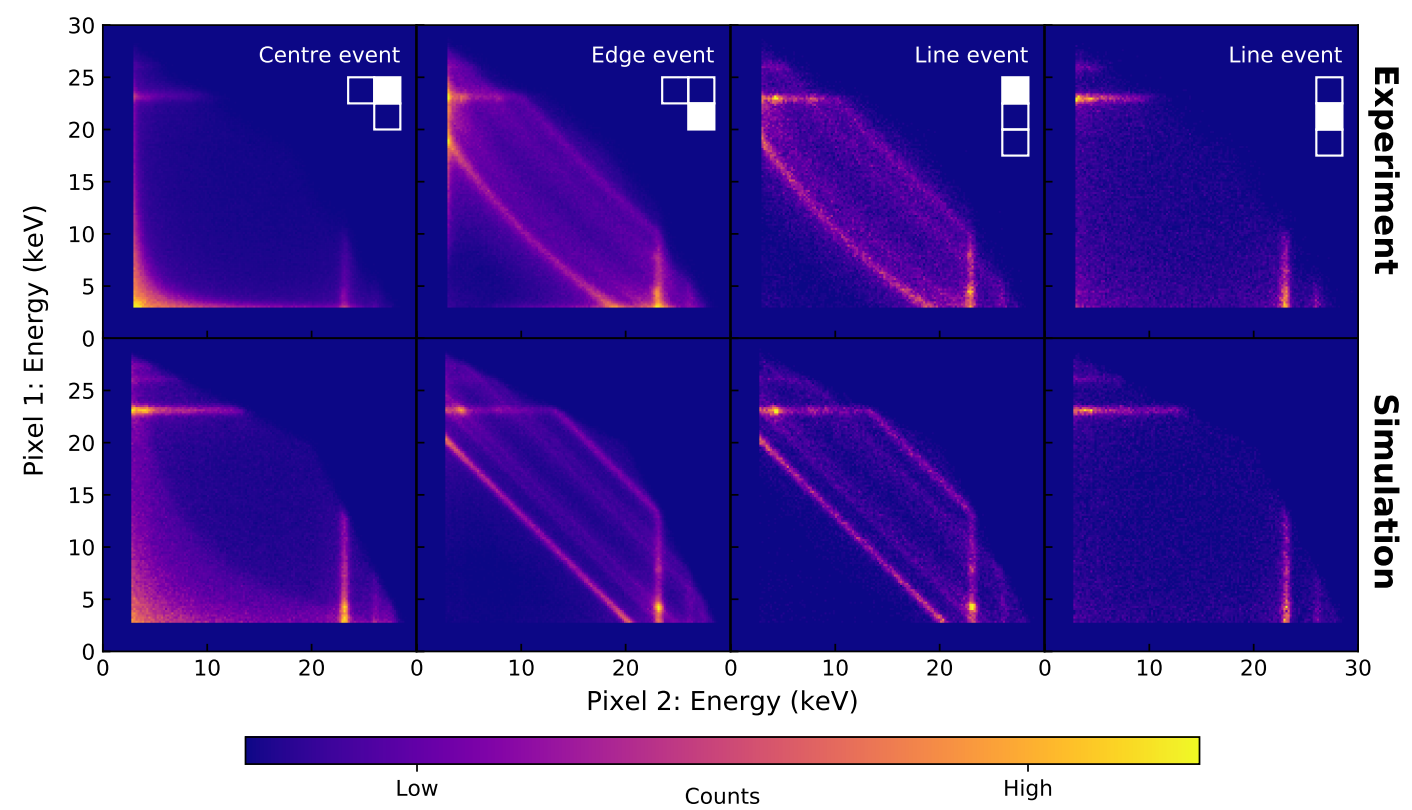

Figure 11: Each image is a distribution of the energy in the two non-maximum pixels from ${ }^{241} \mathrm{Am} 59.5$ $\mathrm{keV}$ tripixel events. The two rows of images correspond to experiment and simulation respectively. Each column of images corresponds to one of the specific tripixel event types - centre, edge, outer line and centre line events. A drawing of the pixels making up the tripixel event type is shown in the first image of each column, with the filled pixel indicating the pixel containing the maximum energy.

The analysis carried out on the bipixels was extended to tripixel events. In order to determine the portion of tripixels due to fluorescence, the tripixels themselves were 
further separated into four types. These types are centre events, where the pixels make a right-angle and the maximum energy is recorded in the centre/middle pixel, edge events, where the pixels also make a right-angle but the maximum energy is recorded in one of the outer end pixels and two types of line events where the three pixels are orientated in a line and the maximum energy is either in an outer or centre pixel. The four tripixel event types are shown in figure 11 - the filled pixel signifies the pixel with the maximum recorded energy.

The majority of tripixel events were found to be in the right-angle arrangement for both the $59.5 \mathrm{keV}$ and $122 \mathrm{keV}$ line energy, at $93.0 \%$ and $91.5 \%$ respectively, with the rest being line events.

The location of the maximum energy pixel in a tripixel event, is an indication of whether charge sharing occurred due to fluorescence or charge spreading. This can be seen in figure 11 which shows the distribution of the energy in the remaining two nonmaximum pixels for each of the four tripixel types. By visual inspection, it is clear the distributions for the edge events and centre events are distinctly different. Edge events are mostly made up of events where summing the energy in the two non-maximum pixels equals the energy of $\mathrm{Cd}(\sim 23 \mathrm{keV})$ or Te $(\sim 26 \mathrm{keV}) \mathrm{K}$-shell fluorescence. For example at the bright streaks in the distribution at 20 and $23 \mathrm{keV}$. Counts along the downwards sloping curve are cases where the energy from the fluorescence photon spreads between the two non-maximum pixels. This leads to charge loss across the pixel gap, giving rise to the non-linear curve as observed previously in figure 7. Edge events therefore appear to be caused by fluorescence, where either an isolated or bipixel event becomes a tripixel due to the fluorescence photon. The distribution for centre events in contrast, shows that most (i.e. $\sim 86 \%$ ) of the events' non-maximum pixels have lower energies around 3-5 keV - well below fluorescence energies. This suggests that centre events are predominately a result of charge spreading over to the adjacent pixels. The centre events distribution shows that some of the events are still due to fluorescence (i.e. the bright streaks), but at lesser rates than for edge events.

The distribution for the outer line events is most alike the edge events distribution, indicating that these events too are generally a consequence of fluorescence. Centre line events occur less frequently, making up $33 \%$ of all line events in the $59.5 \mathrm{keV}$ experimental 
data. Their distribution is more similar to that of right-angle centre events, suggesting more charge spreading in these events, although a larger proportion of counts result from fluorescence, with $\sim 29 \%$ of counts in the fluorescence streaks when using a $1 \mathrm{keV}$ energy window.

\begin{tabular}{ccccc}
\hline & \multicolumn{2}{c}{ Experiment (\%) } & \multicolumn{2}{c}{ Model (\%) } \\
\cline { 2 - 5 } & $59.5 \mathrm{keV}$ & $122 \mathrm{keV}$ & $59.5 \mathrm{keV}$ & $122 \mathrm{keV}$ \\
\hline Bipixels & $12.4 \pm 2.1$ & $9.3 \pm 1.9$ & $11.8 \pm 1.3$ & $8.6 \pm 1.2$ \\
Tripixels & $61.1 \pm 1.1$ & $57.0 \pm 2.5$ & $65.4 \pm 1.5$ & $59.3 \pm 2.0$ \\
\hline
\end{tabular}

Table 7: Percentage of bipixels and tripixels that are due to fluorescence as calculated experimentally and predicted by the model. The uncertainties were calculated from the difference when using a $1 \mathrm{keV}$ and $2 \mathrm{keV}$ energy window around the fluorescence spots/streaks.

By determining all edge and outer line events as resulting from fluorescence, and right-angle and line centre events from charge spreading (with the exception of the bright spots/streaks at 23 and $26 \mathrm{keV}$ ), the fraction of tripixels due to fluorescence was calculated. Using the simulated data at $59.5 \mathrm{keV}$, the same analysis as for the experimental data was performed, also shown in figure 11 for comparison. The distributions from the modelled data show similar results. Since the model does not account for charge loss across the pixel gap, the curves in the edge and outer line event distributions are linear. Curves from the $\mathrm{Cd} \mathrm{K}_{\beta}$ and Te K-shell fluorescence can therefore be resolved. For centre events, the model predicts that the energy shared amongst the non-maximum pixels is less concentrated at lower energies than the experimental data shows. Table 7 shows the portion of tripixels due to fluorescence from both experiment and model. The majority of tripixels appear to be a result of fluorescence, whereas bipixels are predominately due to charge spreading.

The $59.5 \mathrm{keV}$ photopeak for the four different tripixel event types is shown in figure 12. The peak position for the right-angle centre events is lower at $54.5 \mathrm{keV}$ compared to the position for the edge and outer line event peaks at $56.5 \mathrm{keV}$. This again suggests that centre events are mostly due to charge spreading and not fluorescence as they exhibit more charge loss. When removing centre events from the tripixel photopeak, the peak FWHM improves from $5.1 \mathrm{keV}$ to $4.6 \mathrm{keV}$. 


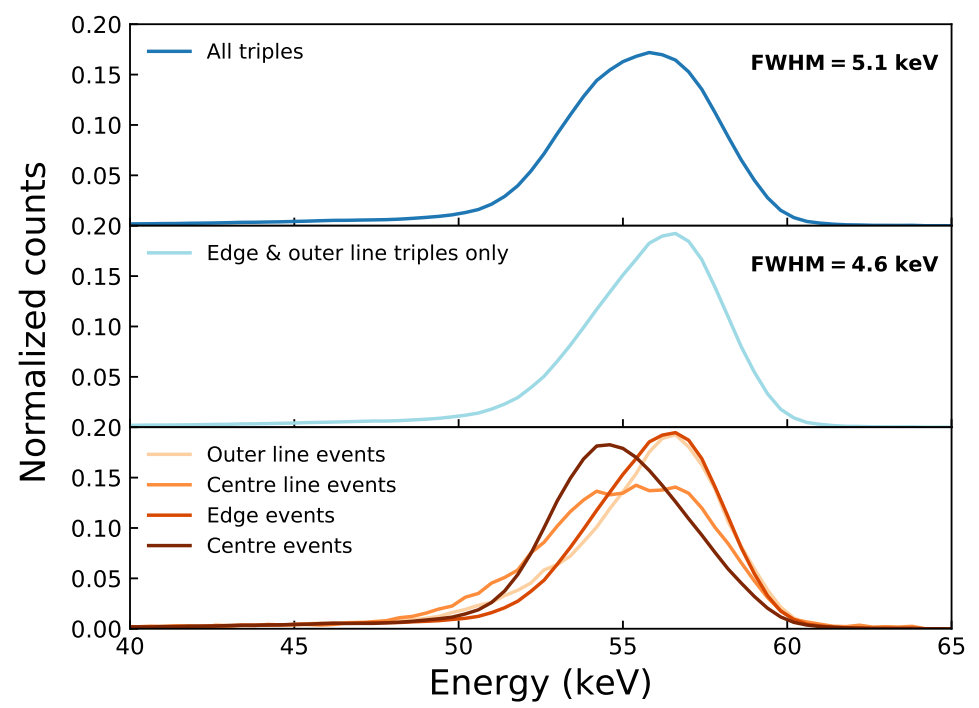

Figure 12: ${ }^{241} \mathrm{Am} 59.5 \mathrm{keV}$ tripixel photopeaks. (Top) photopeak for all tripixel events combined. (Middle) photopeak for outer line and edge tripixel events combined. (Bottom) Individual photopeak for each type of tripixel event. In all cases, the peaks have been normalised as a probability density function.

\section{Conclusion}

This paper described an end-to-end fully spectroscopic Monte-Carlo model which can simulate the spectral response of a pixelated CdTe detector.

The simulated spectra including isolated events only are in good agreement with experimental data taken with a $1 \mathrm{~mm}$ thick CdTe detector coupled to the HEXITEC ASIC, accurately predicting the CdTe escape peaks and fluorescence peaks, and the presence of a low energy tail due to charge trapping and noise thresholding.

High energy pixelated radiation detectors are known to exhibit large amounts of charge sharing which degrades their spectral performance. The model was able to estimate to a good degree the probability of charge sharing occurring at different energies. Given that the model could reproduce results seen experimentally, factors influencing charge sharing could be determined. This showed that the average charge cloud size is in fact relatively constant from $5-100 \mathrm{keV}$ at $\sigma \approx 13 \mu \mathrm{m}$ (a diameter of $\sim 78 \mu \mathrm{m}$ to contain 
$\sim 99.7 \%$ of cloud charges), and that its final size is dominated by diffusion and electron repulsion during drift as opposed to the initial cloud size. The noise threshold therefore significantly reduces the amount of charge sharing at lower photon energies. The CdTe absorption edges were found to have a significant impact on the proportion of shared events.

Fluorescence shared events are characteristically different as they are not a result of charges spreading across pixels, and therefore do not suffer from charge loss to the inter-pixel region. The model was able to predict similar rates for the shared events due to fluorescence as was measured experimentally. Understanding the different types of shared events is critical for certain correction techniques such as the energy-loss correction described in 12, which can currently only correct energy-loss in bipixel events but could be expanded to include tripixels.

The model can be used to estimate the expected probability of charge sharing for different sensor thicknesses, pixel sizes and operating voltages to inform detector design depending on the desired application and energy range of interest. The next steps will be to rigorously test the model with different input parameters when experimental data for comparison is available. The model will also be extended to work with CdZnTe based detectors. The effect of varying the noise threshold on the probability of charge sharing will also be looked at closely.

\section{Acknowledgements}

K.A.L. Koch-Mehrin is supported by a University of Leicester LISEO studentship. The event analysis code is based on work originally carried out by GM Hansford, University of Leicester. This research used the ALICE High Performance Computing Facility at the University of Leicester.

\section{Declaration of competing interest}

The authors declare that there are no known conflicts of interest associated with this publication. 


\section{References}

[1] A. Owens, Compound semiconductor radiation detectors, 1st Edition, Taylor \& Francis Group, European Space Agency, Netherlands, 2012.

[2] P. Seller, S. Bell, R. Cernik, C. Christodoulou, C. Egan, J. Gaskin, S. Jacques, Pixellated $\mathrm{Cd}(\mathrm{Zn})$ Te high-energy x-ray instrument, Journal of Instrumentation 6 (12) (2012) 1-15.

[3] E. Goshen, L. Beilin, E. Stern, Feasibility study of a novel general purpose czt-based digital spect camera: initial clinical results, EJNMMI Phys 14 (2018).

[4] A. Meuris, Semiconductor detector developments for high energy space astronomy, Journal of Instrumentation 9 (2014).

[5] H. Barret, J. Eskin, Charge transport in arrays of semiconductor gamma-ray detectors, Physical Review Letters 75 (1) (1995).

[6] A. Cola, I. Farella, The polarization mechanism in CdTe Schottky detectors, Applied Physics Letters 94 (10) (2009) 1-4.

[7] W. Shockley, Currents to conductors induced by a moving point charge, Journal of Applied Physics 10 (9) (1938) 635.

[8] M. Veale, S. Bell, D. Duarte, A. Schneider, P. Seller, M. D. Wilson, K. Iniewski, Measurements of charge sharing in small pixel cdte detectors, Nuclear Instruments and Methods in Physics Research A 767 (2014) 218-226.

[9] H. Iniewski, Modeling charge-sharing effects in pixellated czt detectors, Nuclear Science Symposium Conference Record 6, 2007.

[10] A. Meuris, O. Limousin, C. Blondel, Charge sharing in cdte pixilated detectors, Nuclear Instruments and Methods in Physics Research A 610 (1) (2009) 294-297.

[11] P. Zambon, V. Radicci, Spectral response characterization of cdte sensors of different pixel size with ibex asic, Nuclear Inst. and Methods in Physics Research A 892 (2018) 106-113. 
[12] S. Bugby, K. Koch-Mehrin, M. Veale, J. Lees, Energy-loss correction in charge sharing events for improved performance of pixellated compound semiconductors, Nuclear Instruments and Methods in Physics Research A 940 (2019) 142-151.

[13] L. Jones, P. Seller, M. Wilson, A. Hardie, Hexitec asic-a pixellated readout chip for czt detectors, Nuclear Instruments and Methods in Physics Research A 604 (1-2) (2009) 34-37.

[14] https://www.python.org/downloads/release/python-370/ (accessed on April 7th, 2020).

[15] R. Evans, The Atomic Nucleus, 1st Edition, McGraw-Hill Book Company, Inc, New York, 1955.

[16] M. Berger, J. Hubbell, S. Seltzer, J. Chang, J. Coursey, R. Sukumar, D. Zucker, K. Olsen, XCOM: Photon cross sections database, NIST, PML, Radiation Physics Division (1998) 87-3597.

[17] M. Born, Atomic Physics, 8th Edition, Blackie and Sons, 1969.

[18] R. Jenkins, Quantitative X-ray Spectrometry, 2nd Edition, Practical Spectroscopy volume 20, Marcel Dekker INC., New York, USA, 1995.

[19] A. Compton, A quantum theory of the scattering of x-rays by light elements, Phys. Rev 21 (5) (1923) 483-502.

[20] O. Klein, Y. Nishina, Über die streuung von strahlung durch freie elektronen nach der neuen relativistischen quantendynamik von dirac, Z. Phys. 52 (1929) 853-868.

[21] A. Thompson, D. Attwood, X-Ray Data booklet, 3rd Edition, Center for X-ray Optics and Advanced Light Source, Berkeley, CA, 2009.

[22] Kaye \& Laby Online, Tables of Physical \& Chemical Constants, https://www. ase.org.uk/resources/kaye-and-laby-tables-physical-constants (accessed on April 4th, 2020).

[23] R. Redus, J. Pantazis, T. Pantazis, A. Huber, B. Cross, Characterization of CdTe detectors for quantitative X-ray spectroscopy, IEEE Transactions on Nuclear Science 56 (4) (2009) 2524-2532. 
[24] M. Benoit, L. Hamel, Simulation of charge collection processes in semiconductor cdznte $\gamma$-ray detectors, Nuclear Instruments and Methods in Physics Research A 606 (3) (2009) 508-516.

[25] P. Trueb, P. Zambon, C. Broennimann, Assessment of the spectral performance of hybrid photon counting x-ray detectors, Medical Physics 44 (9) (2017) 207-214.

[26] A. Fick, Ueber Diffusion, Annalen der Physik (1855).

[27] A. Einstein, Über die von der molekularkinetischen theorie der wärme geforderte bewegung von in ruhenden flüssigkeiten suspendierten teilchen, Annalen der Physik 332 (8) (1901) 549-560.

[28] I. Blevis, R. Levinson, Photon-counting detectors and clinical applications in medical CT imaging, in: J. Iwanczyk (Ed.), Taylor \& Francis Group, Oxford University Press, 2005, Ch. 7, pp. 169-192.

[29] K. Hecht, Zum mechanismus des lichtelektrischen primärstromes in isolierenden kristallen, Zeitschrift für Physik A Hadrons and Nuclei 77 (1932) 235-245.

[30] D. Duarte, J. Lipp, A. Schneider, P. Seller, M. Veale, M. Wilson, M. Baker, P. Sellin, Simulation of active-edge pixelated cdte radiation detectors, Nuclear Instruments and Methods in Physics Research A 806 (2016) 139-145.

[31] M. Veale, S. Bell, P. Seller, M. Wilson, V. Kachkanov, X-ray micro-beam characterization of a small pixel spectroscopic CdTe detector, Journal of Instrumentation 7 (7) (2012).

[32] A. Cola, I. Farella, Electric field properties of CdTe nuclear detectors, IEEE Nuclear Science Symposium Conference Record, 2006.

[33] E. Kalemci, J. L. Matteson, Investigation of charge sharing among electrode strips for a CdZnTe detector, Nuclear Instruments and Methods in Physics Research A: 478 (3) (2002) 527-537.

[34] M. Veale, P. Seller, M. Wilson, E. Liotti, HEXITEC: A High-Energy X-ray Spectroscopic Imaging Detector for Synchrotron Applications, Synchrotron Radiation News 31 (6) (2018) 28-32. 
${ }_{625}$ [35] Amptek, https://www. amptek.com (accessed on April 4th, 2020). 